\title{
GLUCOSE DEPLETION AND DECISION MAKING: AN EXAMINATION OF CHOICE IN CONTEXT
}

Eric Shih, SKK GSB Sungkyunkwan University, Korea Seigyoung Auh, Thunderbird School of Global Management, USA

Bulent Menguc, Brock University, Canada

\begin{abstract}
This paper investigates the idea of an energy model which suggests that the mind requires energy resource when making effortful decisions. In particular, we hypothesize that ingestion of sugar provides the body with glucose as fuel for the brain, and this can reduce reliance on intuitive, heuristic-based decision making. In three experiments, we tested this hypothesis on three types of choices in context, namely reference dependence, attraction, and compromise effect. Participants completed a choice task after drinking lemonade sweetened with either sugar (glucose condition) or Splenda (placebo condition). The results showed that participants who drank lemonade with sugar made more choices that were consistent with using deliberative thinking processes.
\end{abstract}

